\title{
DARK ENERGY FROM COSMIC STRUCTURE
}

\author{
D. L. WILTSHIRE \\ Department of Physics \& Astronomy, University of Canterbury, Private Bag 4800 , \\ Christchurch 8140, New Zealand; and \\ International Center for Relativistic Astrophysics Network (ICRANet), P.le della Repubblica 10, \\ Pescara 65121, Italy \\ E-mail: david.wiltshire@cantebrury.ac.nz
}

\begin{abstract}
Below scales of about 100/h Mpc our universe displays a complex inhomogeneous structure dominated by voids, with clusters of galaxies in sheets and filaments. The coincidence that cosmic expansion appears to start accelerating at the epoch when such structures form has prompted a number of researchers to question whether dark energy is a signature of a failure of the standard cosmology to properly account, on average, for the distribution of matter we observe. Here I discuss the timescape scenario, in which cosmic acceleration is understood as an apparent effect, due to gravitational energy gradients that grow when spatial curvature gradients become significant with the nonlinear growth of cosmic structure. This affects the calibration of local geometry to the solutions of the volume-average evolution equations corrected by backreaction. I further discuss recent work on defining observational tests for average geometric quantities which can distinguish the timescape model from a cosmological constant or other models of dark energy.
\end{abstract}

Keywords: dark energy, theoretical cosmology, observational cosmology

\section{Introduction}

In this paper I will review the conceptual basis ${ }^{1,2}$ and observational $\operatorname{tests}^{3}$ of a cosmology model, ${ }^{1,4}$ which represents a new approach to understanding the phenomenology of dark energy as a consequence of the effect of the growth of inhomogeneous structures. The basic idea, outlined in a nontechnical manner in ref. [5], is that as inhomogeneities grow one must consider not only their backreaction on average cosmic evolution, but also the variance in the geometry as it affects the calibration of clocks and rulers of ideal observers. Dark energy is then effectively realised as a misidentification of gravitational energy gradients.

Although the standard Lambda Cold Dark Matter $(\Lambda \mathrm{CDM})$ model provides a good fit to many tests, there are tensions between some tests, and also a number of puzzles and anomalies. Furthermore, at the present epoch the observed universe is only statistically homogeneous once one samples on scales of 150-300 Mpc. Below such scales it displays a web-like structure, dominated in volume by voids. Some $40 \%-50 \%$ of the volume of the present epoch universe is in voids with $\delta \rho / \rho \sim-1$ on scales of $30 h^{-1} \mathrm{Mpc}^{6}$ where $h$ is the dimensionless parameter related to the Hubble constant by $H_{0}=100 h \mathrm{~km} \mathrm{sec} \mathrm{kpc}^{-1}$. Once one also accounts for numerous minivoids, and perhaps also a few larger voids, then it appears that the present epoch universe is void-dominated. Clusters of galaxies are spread in sheets that surround these voids, and in thin filaments that thread them.

A number of different approaches have been taken to study inhomogeneous cosmologies. One large area of research is that of exact solutions of Einstein's equations 
(see, e.g., ref. [7]), and of the Lemaître-Tolman-Bondi ${ }^{8}$ (LTB) dust solution in particular. While one mimic any luminosity distance relation with LTB models, generally the inhomogeneities required to match the supernova data are much larger than the typical scales of voids described above. Furthermore, one must assume the unlikely symmetry of a spherically symmetric universe about our point, which violates the Copernican principle. It is my view that while the LTB solutions are interesting toy models, one should retain the Copernican principle in a statistical sense, and one should seriously try to model the universe with those scales of inhomogeneity that we observe.

One particular consequence of a matter distribution that is only statistically homogeneous, rather than exactly homogeneous, is that when the Einstein equations are averaged they do not evolve as a smooth Friedmann-Lemaitre-RobertsonWalker (FLRW) geometry. Instead the Friedmann equations are supplemented by additional backreaction terms ${ }^{\mathrm{a}} .{ }^{10}$ Whether or not one can fully explain the expansion history of the universe as a consequence of the growth of inhomogeneities and backreaction, without a fluid-like dark energy, is the subject of ongoing debate. ${ }^{11}$

A typical line of reasoning against backreaction is that of a plausibility argument: ${ }^{12}$ if we assume a FLRW geometry with small perturbations, and estimate the magnitude of the perturbations from the typical rotational and peculiar velocities of galaxies, then the corrections of inhomogeneities are consistently small. This would be a powerful argument, were it not for the fact that at the present epoch galaxies are not homogeneously distributed. The Hubble Deep Field reveals that galaxies were close to being homogeneous distributed at early epochs, but following the growth voids at redshifts $z \lesssim 1$ that is no longer the case today. Therefore galaxies cannot be consistently treated as randomly distributed gas particles on the $30 h^{-1}$ Mpc scales ${ }^{6}$ that dominate present cosmic structure below the scale of statistical homogeneity.

Over the past few years I have developed a new physical interpretation of cosmological solutions within the Buchert averaging scheme. ${ }^{1,2,4}$ I start by noting that in the presence of strong spatial curvature gradients, not only should the average evolution equations be replaced by equations with terms involving backreaction, but the physical interpretation of average quantities must also account for the differences between the local geometry and the average geometry. In other words, geometric variance can be just as important as geometric averaging when it comes to the physical interpretation of the expansion history of the universe.

I proceed from the fact that structure formation provides a natural division of scales in the observed universe. As observers in galaxies, we and the objects we observe in other galaxies are necessarily in bound structures, which formed from density perturbations that were greater than critical density. If we consider the evidence of the large scale structure surveys on the other hand, then the average

${ }^{\mathrm{a}}$ For a general review of averaging and backreaction see, e.g., the article by van den Hoogen in this volume. ${ }^{9}$ 
location by volume in the present epoch universe is in a void, which is negatively curved. We can expect systematic differences in spatial curvature between the average mass environment, in bound structures, and the volume-average environment, in voids.

Spatial curvature gradients will in general give rise to gravitational energy gradients, and herein lie the issue which I believe are key to understanding the phenomenon of dark energy. The definition of gravitational energy in general relativity is notoriously subtle. This is due to the equivalence principle, which means that we can always get rid of gravity near a point. As a consequence, the energy, momentum and angular momentum associated with the gravitational field, which have macroscopic effects on the relative calibrations of the clocks and rulers of observers, cannot be described by local quantities encoded in a fluidlike energy-momentum tensor. Instead they are at best quasi-local. ${ }^{13}$ There is no general agreement on how to deal with quasi-local gravitational energy. It is my view that since the issue has its origin in the equivalence principle, we must return to first principles and reconsider the equivalence principle in the context of cosmological averages.

\section{The cosmological equivalence principle}

In laying the foundations of general relativity, Einstein sought to refine our physical understanding of that most central physical concept: inertia. As he stated: "In a consistent theory of relativity there can be be no inertia relatively to 'space', but only an inertia of masses relatively to one another". ${ }^{14}$ This is the general philosophy that underlies Mach's principle, which strongly guided Einstein. However, the refinement of the understanding of inertia that Einstein left us with in relation to gravity, the Strong Equivalence Principle (SEP), only goes part-way in addressing Mach's principle.

Mach's principle may be stated:15,16 "Local inertial frames (LIFs) are determined through the distributions of energy and momentum in the universe by some weighted average of the apparent motions". The SEP says nothing about the average effect of gravity, and therefore nothing about the "suitable weighted average of the apparent motions" of the matter in the universe. Since gravity for ordinary matter fields obeying the strong energy condition is universally attractive, the spacetime geometry of a universe containing matter is not stable, but is necessarily dynamically evolving. Therefore, accounting for the average effect of matter to address Mach's principle means that any relevant frame in cosmological averages is one in which time symmetries of the Lorentz group in LIFs are removed.

My proposal for applying the equivalence principle on cosmological scales is to deal with the average effects of the evolving density by extending the SEP to larger regional frames while removing the time translation and boost symmetries of the LIF to define a Cosmological Equivalence Principle as follows: ${ }^{2}$

At any event, always and everywhere, it is possible to choose a suitably defined spacetime neighbourhood, the cosmological inertial frame (CIF), in which average 
motions (timelike and null) can be described by geodesics in a geometry that is Minkowski up to some time-dependent conformal transformation,

$$
\mathrm{d} s_{\mathrm{CIF}}^{2}=a^{2}(\eta)\left[-\mathrm{d} \eta^{2}+\mathrm{d} r^{2}+r^{2}\left(\mathrm{~d} \theta^{2}+\sin ^{2} \theta \mathrm{d} \phi^{2}\right)\right]
$$

Since the average geometry is a time-dependent conformal scaling of Minkowski space, the CEP reduces to the standard SEP if $a(\eta)$ is constant, or alternatively over very short time intervals during which the time variation of $a(\eta)$ can be neglected. The relation to cosmological averages is understood by the fact that (1) is the spatially flat FLRW metric. In the standard cosmology this is taken to be the geometry of the whole universe. Here, however, the whole universe is inhomogeneous but its geometry is restricted by the requirement that it is possible to always choose (1) as a regional average. This would rule out geometries with global anisotropies, such as Bianchi models, while hopefully leaving enough room to describe an inhomogeneous but statistically homogeneous universe like the one we observe.

To understand why an average geometry (1) is a relevant average reference geometry for the relative calibration of rulers and clocks in the absence of global Killing vectors, let us construct what I will call the semi-tethered lattice by the following means. Take a lattice of observers in Minkowski space, initially moving isotropically away from each nearest neighbour at uniform initial velocities. The lattice of observers are chosen to be equidistant along mutual oriented $\hat{x}, \hat{y}$ and $\hat{z}$ axes. Now suppose that the observers are each connected to six others by strings of negligible mass and identical tension along the mutually oriented spatial axes. The strings are not fixed but unwind freely from spools on which an arbitrarily long supply of string is wound. The strings initially unreel at the same uniform rate, representing a "recession velocity". Each observer carries synchronised clocks, and at a prearranged local proper time all observers apply brakes to each spool, the braking mechanisms having been pre-programmed to deliver the same impulse as a function of local time.

The semi-tethered lattice experiment is directly analogous to the decelerating volume expansion of (1) due to some average homogeneous matter density, because it maintains the homogeneity and isotropy of space over a region as large as the lattice. Work is done in applying the brakes, and energy can be extracted from this - just as kinetic energy of expansion of the universe is converted to other forms by gravitational collapse. Since brakes are applied in unison, however, there is no net force on any observer in the lattice, justifying the inertial frame interpretation, even though each observer has a non-zero 4-acceleration with respect to the global Minkowski frame. The braking function may have an arbitrary time profile; provided it is applied uniformly at every lattice site the clocks will remain synchronous in the comoving sense, as all observers have undergone the same relative deceleration.

Let us now consider two sets of disjoint semi-tethered lattices, with identical initial local expansion velocities, in a background static Minkowski space. (See Fig. 1(a).) Observers in the first congruence apply brakes in unison to decelerate homogeneously and isotropically at one rate. Observers in the second congru- 
ence do so similarly, but at a different rate. Suppose that when transformed to a global Minkowski frame, with time $t$, that at each time step the magnitudes of the 4 -decelerations satisfy $\alpha_{1}(t)>\alpha_{2}(t)$ for the respective congruences. By special relativity, since members of the first congruence decelerate more than those of the second congruence, at any time $t$ their proper times satisfy $\tau_{1}<\tau_{2}$. The members of the first congruence age less quickly than members of the second congruence.
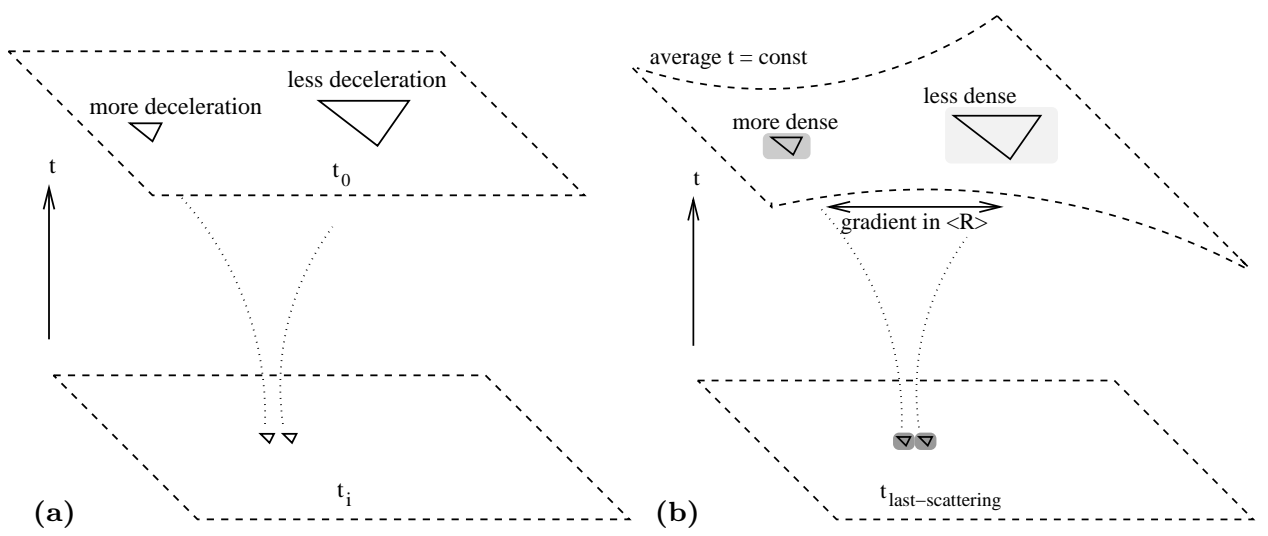

Fig. 1. Two equivalent situations: (a) in Minkowski space observers in separate semi-tethered lattices, initially expanding at the same rate, apply brakes homogeneously and isotropically within their respective regions but at different rates; (b) in the universe which is close to homogeneous and isotropic at last-scattering comoving observers in separated regions initially move away from each other isotropically, but experience different locally homogeneous isotropic decelerations as local density contrasts grow. In both cases there is a relative deceleration of the observer congruences and those in the region which has decelerated more will age less.

By the CEP, the case of volume expansion of two disjoint regions of different average density in the actual universe is entirely analogous. The equivalence of the circumstance rests on the fact that the expansion of the universe was extremely uniform at the time of last scattering, by the evidence of the CMB. At that epoch all regions had almost the same density - with tiny fluctuations - and the same uniform Hubble flow. At late epochs, suppose that in the frame of any average cosmological observer there are expanding regions of different density which have decelerated by different amounts by a given time, $t$, according to that observer. Then by the CEP the local proper time of the comoving observers in the denser region, which has decelerated more, will be less than that of the equivalent observers in the less dense region which has decelerated less. (See Fig. 1(b).) Consequently the proper time of the observers in the more dense CIF will be less than that of those in the less dense CIF, by equivalence of the two situations.

The fact that a global Minkowski observer does not exist in the second case does not invalidate the argument. The global Minkowski time is just a coordinate label. In the cosmological case the only restriction is that the expansion of both average congruences must remain homogeneous and isotropic in local regions of 
different average density in the global average $t=$ const slice. Provided we can patch the regional frames together suitably, then if regions in such a slice are still expanding and have a significant density contrast we can expect a significant clock rate variance.

This equivalence directly establishes the idea of a gravitational energy cost for a spatial curvature gradient, since the existence of expanding regions of different density within an average $t=$ const slice implies a gradient in the average Ricci scalar curvature, $\langle\mathcal{R}\rangle$, on one hand, while the fact that the local proper time varies on account of the relative deceleration implies a gradient in gravitational energy on the other.

In the actual universe, the question is: can the effect described above be significant enough to give a significant variation in the clocks of ideal isotropic observers (those who see an isotropic mean $\mathrm{CMB}$ ) in regions of different density, who experience a relative deceleration of their regional volume expansions? Since we are dealing with weak fields the relative deceleration of the background is small. Nonetheless even if the relative deceleration is typically of order $10^{-10} \mathrm{~ms}^{-2}$, cumulatively over the age of the universe it leads to significant clock rate variances, ${ }^{2}$ of the order of $38 \%$. Such a large effect is counterintuitive, as we are used to only considering time dilations due to relative accelerations within the static potentials of isolated systems. Essentially, we are dealing with a different physical effect concerning the relative synchronization of clocks in the absence of global Killing vectors. A small instantaneous relative deceleration can lead to cumulatively large differences, given one has the lifetime of the universe to play with. As a consequence the age of the universe itself becomes position-dependent. Since we and all the objects we observe are necessarily in regions of greater than critical density, on account of structure formation we have a mass-biased view of the universe and cannot directly observe such variations.

\section{A detailed cosmological model}

I proceed from an ansatz that the variance in gravitational energy is correlated with the average spatial curvature in such a way as to implicitly solve the Sandagede Vaucouleurs paradox that a statistically quiet, broadly isotropic, Hubble flow is observed deep below the scale of statistical homogeneity. In particular, galaxy peculiar velocities have a small magnitude with respect to a local regional volume expansion. Expanding regions of different densities are patched together so that the regionally measured expansion remains uniform. Such regional expansion refers to the variation of the regional proper length, $\ell_{r}=\mathcal{V}^{1 / 3}$, with respect to proper time of isotropic observers Although voids open up faster, so that their proper volume increases more quickly, on account of gravitational energy gradients the local clocks will also tick faster in a compensating manner.

Details of the fitting of local observables to average quantities for solutions to the Buchert formalism are described in detail in refs. [1,4]. Negatively curved voids, 
and spatially flat expanding wall regions within which galaxy clusters are located, are combined in a Buchert average

$$
f_{\mathrm{v}}(t)+f_{\mathrm{w}}(t)=1
$$

where $f_{\mathrm{w}}(t)=f_{\mathrm{wi}} a_{\mathrm{w}}^{3} / \bar{a}^{3}$ is the wall volume fraction and $f_{\mathrm{v}}(t)=f_{\mathrm{vi}} a_{\mathrm{v}}^{3} / \bar{a}^{3}$ is the void volume fraction, $\mathcal{V}=\mathcal{V}_{\mathrm{i}} \bar{a}^{3}$ being the present horizon volume, and $f_{\mathrm{wi}}, f_{\mathrm{vi}}$ and $\mathcal{V}_{\mathrm{i}}$ initial values at last scattering. The time parameter, $t$, is the volume-average time parameter of the Buchert formalism, but does not coincide with that of local measurements in galaxies. In trying to fit a FLRW solution to the universe we attempt to match our local spatially flat wall geometry

$$
\mathrm{d} s_{f i}^{2}=-\mathrm{d} \tau^{2}+a_{\mathrm{w}}^{2}(\tau)\left[\mathrm{d} \eta_{\mathrm{w}}^{2}+\eta_{\mathrm{w}}^{2} \mathrm{~d} \Omega^{2}\right] .
$$

to the whole universe, when in reality the calibration of rulers and clocks of ideal isotropic observers vary with gradients in spatial curvature and gravitational energy. By conformally matching radial null geodesics with those of the Buchert average solutions, the geometry (3) may be extended to cosmological scales as the dressed geometry

$$
\mathrm{d} s^{2}=-\mathrm{d} \tau^{2}+a^{2}(\tau)\left[\mathrm{d} \bar{\eta}^{2}+r_{\mathrm{w}}^{2}(\bar{\eta}, \tau) \mathrm{d} \Omega^{2}\right]
$$

where $a=\bar{\gamma}^{-1} \bar{a}, \bar{\gamma}=\frac{\mathrm{d} t}{\mathrm{~d} \tau}$ is the relative lapse function between wall clocks and volume-average ones, $\mathrm{d} \bar{\eta}=\mathrm{d} t / \bar{a}=\mathrm{d} \tau / a$, and $r_{\mathrm{w}}=\bar{\gamma}\left(1-f_{\mathrm{v}}\right)^{1 / 3} f_{\mathrm{wi}}{ }^{-1 / 3} \eta_{\mathrm{w}}(\bar{\eta}, \tau)$, where $\eta_{\mathrm{w}}$ is given by integrating $\mathrm{d} \eta_{\mathrm{w}}=f_{\mathrm{wi}}{ }^{1 / 3} \mathrm{~d} \bar{\eta} /\left[\bar{\gamma}\left(1-f_{\mathrm{v}}\right)^{1 / 3}\right]$ along null geodesics.

In addition to the bare cosmological parameters which describe the Buchert equations, one obtains dressed parameters relative to the geometry (4). For example, the dressed matter density parameter is $\Omega_{M}=\bar{\gamma}^{3} \bar{\Omega}_{M}$, where $\bar{\Omega}_{M}=$ $8 \pi G \bar{\rho}_{M 0} \bar{a}_{0}^{3} /\left(3 \bar{H}^{2} \bar{a}^{3}\right)$ is the bare matter density parameter. The dressed parameters take numerical values close to the ones inferred in standard FLRW models.

\subsection{Apparent acceleration and Hubble flow variance}

The gradient in gravitational energy and cumulative differences of clock rates between wall observers and volume average ones has important physical consequences. Using the exact solution obtained in ref. [4], one finds that a volume average observer would infer an effective deceleration parameter $\bar{q}=-\ddot{\bar{a}} /\left(\bar{H}^{2} \bar{a}\right)=$ $2\left(1-f_{\mathrm{v}}\right)^{2} /\left(2+f_{\mathrm{v}}\right)^{2}$, which is always positive since there is no global acceleration. However, a wall observer infers a dressed deceleration parameter

$$
q=-\frac{1}{H^{2} a} \frac{\mathrm{d}^{2} a}{\mathrm{~d} \tau^{2}}=\frac{-\left(1-f_{\mathrm{v}}\right)\left(8 f_{\mathrm{v}}^{3}+39 f_{\mathrm{v}}^{2}-12 f_{\mathrm{v}}-8\right)}{\left(4+f_{\mathrm{v}}+4 f_{\mathrm{v}}{ }^{2}\right)^{2}},
$$

where the dressed Hubble parameter is given by

$$
H=a^{-1} \frac{\mathrm{d}}{\mathrm{d} \tau} a=\bar{\gamma} \bar{H}-\dot{\bar{\gamma}}=\bar{\gamma} \bar{H}-\bar{\gamma}^{-1} \frac{\mathrm{d}}{\mathrm{d} \tau} \bar{\gamma} .
$$

At early times when $f_{\mathrm{v}} \rightarrow 0$ the dressed and bare deceleration parameter both take the Einstein-de Sitter value $q \simeq \bar{q} \simeq \frac{1}{2}$. However, unlike the bare parameter which 
monotonically decreases to zero, the dressed parameter becomes negative when $f_{\mathrm{v}} \simeq 0.59$ and $\bar{q} \rightarrow 0^{-}$at late times. For the best-fit parameters ${ }^{17}$ the apparent acceleration begins at a redshift $z \simeq 0.9$.

Cosmic acceleration is thus revealed as an apparent effect which arises due to the cumulative clock rate variance of wall observers relative to volume-average observers. It becomes significant only when the voids begin to dominate the universe by volume. Since the epoch of onset of apparent acceleration is directly related to the void fraction, $f_{\mathrm{v}}$, this solves one cosmic coincidence problem.

In addition to apparent cosmic acceleration, a second important apparent effect will arise if one considers scales below that of statistical homogeneity. By any one set of clocks it will appear that voids expand faster than wall regions. Thus a wall observer will see galaxies on the far side of a dominant void of diameter $30 h^{-1}$ Mpc recede at a rate greater than the dressed global average $H_{0}$, while galaxies within an ideal wall will recede at a rate less than $H_{0}$. Since the uniform bare rate $\bar{H}$ would also be the local value within an ideal wall, eq. (6) gives a measure of the variance in the apparent Hubble flow. The best-fit parameters ${ }^{17}$ give a dressed Hubble constant $H_{0}=61.7_{-1.1}^{+1.2} \mathrm{~km} \mathrm{sec}^{-1} \mathrm{Mpc}^{-1}$, and a bare Hubble constant $\bar{H}_{0}=$ $48.2_{-2.4}^{+2.0} \mathrm{~km} \mathrm{sec}^{-1} \mathrm{Mpc}^{-1}$. The present epoch variance is $17-22 \%$.

Since voids dominate the universe by volume at the present epoch, any observer in a galaxy in a typical wall region will measure locally higher values of the Hubble constant, with peak values of order $72 \mathrm{~km} \mathrm{sec}^{-1} \mathrm{Mpc}^{-1}$ at the $30 h^{-1} \mathrm{Mpc}$ scale of the dominant voids. Over larger distances, as the line of sight intersects more walls as well as voids, a radial spherically symmetric average will give an average Hubble constant whose value decreases from the maximum at the $30 h^{-1} \mathrm{Mpc}$ scale to the dressed global average value, as the scale of homogeneity is approached at roughly the baryon acoustic oscillation (BAO) scale of $110 h^{-1} \mathrm{Mpc}$. This predicted effect could account for the Hubble bubble ${ }^{18}$ and more detailed studies of the scale dependence of the local Hubble flow. ${ }^{19}$

In fact, the variance of the local Hubble flow below the scale of homogeneity should correlate strongly to observed structures in a manner which has no equivalent prediction in FLRW models.

\section{Future observational tests}

There are two types of potential cosmological tests that can be developed; those relating to scales below that of statistical homogeneity as discussed above, and those that relate to averages on our past light cone on scales much greater than the scale of statistical homogeneity. The second class of tests includes equivalents to all the standard cosmological tests of the standard FLRW model with Newtonian perturbations. This second class of tests can be further divided into tests which just deal with the bulk cosmological averages (luminosity and angular diameter distances etc), and those that deal with the variance from the growth of structures (late epoch integrated Sachs-Wolfe effect, cosmic shear, redshift space distortions 
etc). Here I will concentrate solely on the simplest tests which are directly related to luminosity and angular diameter distance measures.

In the timescape cosmology we have an effective dressed luminosity distance

$$
d_{L}=a_{0}(1+z) r_{\mathrm{w}}
$$

where $a_{0}=\bar{\gamma}_{0}^{-1} \bar{a}_{0}$, and

$$
r_{\mathrm{w}}=\bar{\gamma}\left(1-f_{\mathrm{v}}\right)^{1 / 3} \int_{t}^{t_{0}} \frac{\mathrm{d} t^{\prime}}{\bar{\gamma}\left(t^{\prime}\right)\left(1-f_{\mathrm{v}}\left(t^{\prime}\right)\right)^{1 / 3} \bar{a}\left(t^{\prime}\right)} .
$$

We can also define an effective angular diameter distance, $d_{A}$, and an effective comoving distance, $D$, to a redshift $z$ in the standard fashion

$$
d_{A}=\frac{D}{1+z}=\frac{d_{L}}{(1+z)^{2}}
$$

A direct method of comparing the distance measures with those of homogeneous models with dark energy, is to observe that for a standard spatially flat cosmology with dark energy obeying an equation of state $P_{D}=w(z) \rho_{D}$, the quantity

$$
H_{0} D=\int_{0}^{z} \frac{\mathrm{d} z^{\prime}}{\sqrt{\Omega_{M 0}\left(1+z^{\prime}\right)^{3}+\Omega_{D 0} \exp \left[3 \int_{0}^{z^{\prime}} \frac{\left(1+w\left(z^{\prime \prime}\right)\right) \mathrm{d} z^{\prime \prime}}{1+z^{\prime \prime}}\right]}},
$$

does not depend on the value of the Hubble constant, $H_{0}$, but only directly on $\Omega_{M 0}=1-\Omega_{D 0}$. Since the best-fit values of $H_{0}$ are potentially different for the different scenarios, a comparison of $H_{0} D$ curves as a function of redshift for the timescape model versus the $\Lambda \mathrm{CDM}$ model gives a good indication of where the largest differences can be expected, independently of the value of $H_{0}$. Such a comparison is made in Fig. 2.

We see that as redshift increases the timescape model interpolates between $\Lambda \mathrm{CDM}$ models with different values of $\Omega_{M 0}$. For redshifts $z \lesssim 1.5 D_{\mathrm{TS}}$ is very close to $D_{\Lambda \mathrm{CDM}}$ for the parameter values $\left(\Omega_{M 0}, \Omega_{\Lambda 0}\right)=(0.34,0.66)$ (model (iii)) which best-fit the Riess07 supernovae (SneIa) data ${ }^{21}$ only, by our own analysis. For very large redshifts that approach the surface of last scattering, $z \lesssim 1100$, on the other hand, $D_{\mathrm{TS}}$ very closely matches $D_{\Lambda \mathrm{CDM}}$ for the parameter values $\left(\Omega_{M 0}, \Omega_{\Lambda 0}\right)=(0.249,0.751)$ (model $\left.(\mathrm{i})\right)$ which best-fit WMAP5 only. ${ }^{20}$ Over redshifts $2 \lesssim z \lesssim 10$, at which scales independent tests are conceivable, $D_{\text {TS }}$ makes a transition over corresponding curves of $D_{\Lambda \mathrm{CDM}}$ with intermediate values of $\left(\Omega_{M 0}, \Omega_{\Lambda 0}\right)$. The $D_{\Lambda \mathrm{CDM}}$ curve for joint best-fit parameters to SneIa, BAO measurements and WMAP $5,{ }^{20}\left(\Omega_{M 0}, \Omega_{\Lambda 0}\right)=(0.279,0.721)$ is best-matched over the range $5 \lesssim z \lesssim 6$, for example.

The difference of $D_{\mathrm{TS}}$ from any single $D_{\Lambda \mathrm{CDM}}$ curve is perhaps most pronounced in the range $2 \lesssim z \lesssim 6$, which may be an optimal regime to probe in future experiments. Gamma-ray bursters (GRBs) now probe distances to redshifts $z \lesssim 8.3$, and could be very useful. A considerable amount work of work has already been done on Hubble diagrams for GRBs. (See, e.g., ${ }^{22}$ ) Much more work is needed to nail 


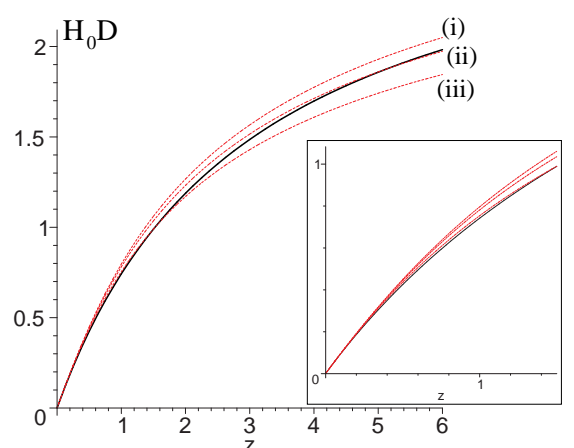

(a)

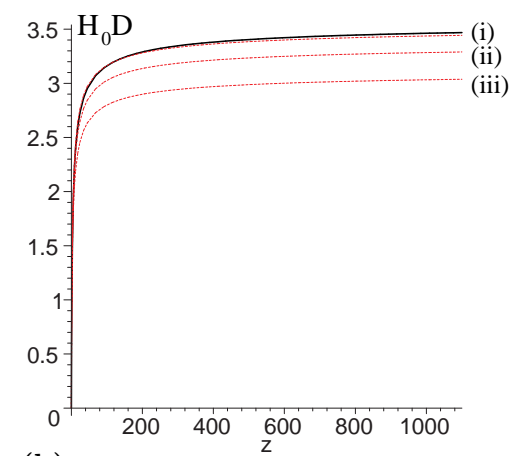

(b)

Fig. 2. The effective comoving distance $H_{0} D(z)$ is plotted for the best-fit timescape (TS) model, with $f_{\mathrm{v} 0}=0.762$, (solid line); and for various spatially flat $\Lambda$ CDM models (dashed lines). The parameters for the dashed lines are (i) $\Omega_{M 0}=0.249$ (best-fit to WMAP5 only ${ }^{20}$ ); (ii) $\Omega_{M 0}=0.279$ (joint best-fit to SneIa, BAO and WMAP5); (iii) $\Omega_{M 0}=0.34$ (best-fit to Riess07 Snela only ${ }^{21}$ ). Panel (a) shows the redshift range $z<6$, with an inset for $z<1.5$, which is the range tested by current SneIa data. Panel (b) shows the range $z<1100$ up to the surface of last scattering, tested by WMAP.

down systematic uncertainties, but GRBs may eventually provide a definitive test in future. An analysis of the timescape model Hubble diagram using 69 GRBs has just been performed by Schaefer, ${ }^{23}$ who finds that it fits the data better than the concordance $\Lambda \mathrm{CDM}$ model, but not yet by a huge margin. As more data is accumulated, it should become possible to distinguish the models if the issues with the standardization of GRBs can be ironed out.

\subsection{The effective "equation of state"}

It should be noted that the shape of the $H_{0} D$ curves depicted in Fig. 2 represent the observable quantity one is actually measuring when some researchers loosely talk about "measuring the equation of state". For spatially flat dark energy models, with $H_{0} D$ given by (10), one finds that the function $w(z)$ appearing in the fluid equation of state $P_{D}=w(z) \rho_{D}$ is related to the first and second derivatives of (10) by

$$
w(z)=\frac{\frac{2}{3}(1+z) D^{\prime-1} D^{\prime \prime}+1}{\Omega_{M 0}(1+z)^{3} H_{0}^{2} D^{\prime 2}-1}
$$

where prime denotes a derivative with respect to $z$. Such a relation can be applied to observed distance measurements, regardless of whether the underlying cosmology has dark energy or not. Since it involves first and second derivatives of the observed quantities, it is actually much more difficult to determine observationally than directly fitting $H_{0} D(z)$.

The equivalent of the "equation of state", $w(z)$, for the timescape model is plotted in Fig. 3. The fact that $w(z)$ is undefined at a particular redshift and changes sign through $\pm \infty$ simply reflects the fact that in (11) we are dividing by a 
quantity which goes to zero for the timescape model, even though the underlying curve of Fig. 2 is smooth. Since one is not dealing with a dark energy fluid in the present case, $w(z)$ simply has no physical meaning. Nonetheless, phenomenologically the results do agree with the usual inferences about $w(z)$ for fits of standard dark energy cosmologies to SneIa data. For the canonical model of Fig. 3(a) one finds that the average value of $w(z) \simeq-1$ on the range $z \lesssim 0.7$, while the average value of $w(z)<-1$ if the range of redshifts is extended to higher values. The $w=-1$ "phantom divide" is crossed at $z \simeq 0.46$ for $f_{\mathrm{v} 0} \simeq 0.76$. One recent study ${ }^{24}$ finds mild $95 \%$ evidence for an equation of state that crosses the phantom divide from $w>-1$ to $w<-1$ in the range $0.25<z<0.75$ in accord with the timescape expectation. By contrast, another study ${ }^{25}$ at redshifts $z<1$ draws different conclusions about dynamical dark energy, but for the given uncertainties in $w(z)$ the data is consistent with Fig. 2(a) as well as with a cosmological constant. ${ }^{3}$

The fact that $w(z)$ is a different sign to the dark energy case for $z>2$ is another way of viewing our statement above that the redshift range $2 \lesssim z \lesssim 6$ may be optimal for discriminating model differences.
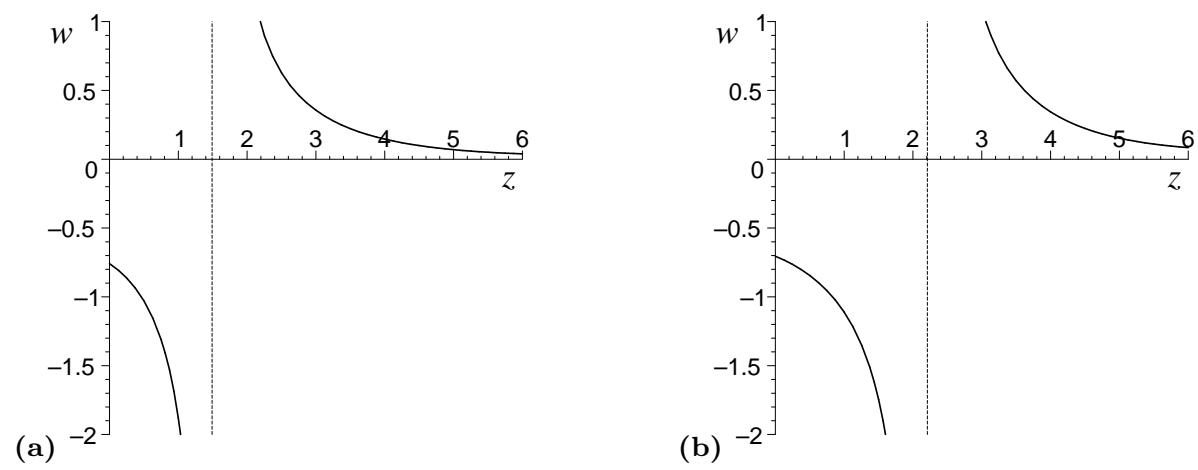

Fig. 3. The artificial equivalent of an equation of state constructed using the effective comoving distance (11), plotted for the timescape tracker solution with best-fit value $f_{\mathrm{v} 0}=0.762$, and two different values of $\Omega_{M 0}:$ (a) the canonical dressed value $\Omega_{M 0}=\frac{1}{2}\left(1-f_{\mathrm{v} 0}\right)\left(2+f_{\mathrm{v} 0}\right)=0.33 ;(\mathbf{b})$ $\Omega_{M 0}=0.279$.

\subsection{The $H(z)$ measure}

Further observational diagnostics can be devised if the expansion rate $H(z)$ can be observationally determined as a function of redshift. Recently such a determination of $H(z)$ at $z=0.24$ and $z=0.43$ has been made using redshift space distortions of the BAO scale in the $\Lambda$ CDM model. ${ }^{26}$ This technique is of course model dependent, and the Kaiser effect would have to be re-examined in the timescape model before a direct comparison of observational results could be made. A model-independent measure of $H(z)$, the redshift time drift test, is discussed below.

In Fig. 4 we compare $H(z) / H_{0}$ for the timescape model to spatially flat $\Lambda$ CDM 
models with the same parameters chosen in Fig. 2. The most notable feature is that the slope of $H(z) / H_{0}$ is less than in the $\Lambda$ CDM case, as is to be expected for a model whose (dressed) deceleration parameter varies more slowly than for $\Lambda$ CDM.

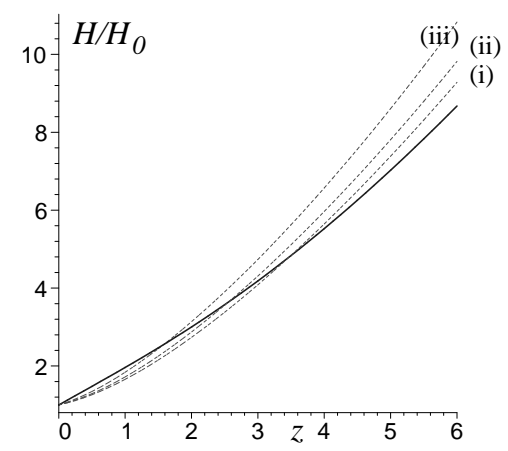

Fig. 4. The function $H_{0}^{-1} H(z)$ for the timescape model with $f_{\mathrm{v} 0}=0.762$ (solid line) is compared to $H_{0}^{-1} H(z)$ for three spatially flat $\Lambda \mathrm{CDM}$ models with the same values of $\left(\Omega_{M 0}, \Omega_{\Lambda 0}\right)$ as in Fig. 2 (dashed lines).

\subsection{The $\mathrm{Om}(z)$ measure}

Recently a number of authors ${ }^{27-29}$ have discussed various roughly equivalent diagnostics of dark energy. For example, Sahni, Shafieloo and Starobinsky, ${ }^{28}$ have proposed a diagnostic function

$$
O m(z)=\left[\frac{H^{2}(z)}{H_{0}^{2}}-1\right]\left[(1+z)^{3}-1\right]^{-1},
$$

on account of the fact that it is equal to the constant present epoch matter density parameter, $\Omega_{M 0}$, at all redshifts for a spatially flat FLRW model with pressureless dust and a cosmological constant. However, it is not constant if the cosmological constant is replaced by other forms of dark energy. For general FLRW models, $H(z)=\left[D^{\prime}(z)\right]^{-1} \sqrt{1+\Omega_{k 0} H_{0}^{2} D^{2}(z)}$, which only involves a single derivatives of $D(z)$. Thus the diagnostic (12) is easier to reconstruct observationally than the equation of state parameter, $w(z)$.

The quantity $\mathrm{Om}(z)$ is readily calculated for the timescape model, and the result is displayed in Fig. 5. What is striking about Fig. 5, as compared to the curves for quintessence and phantom dark energy models as plotted in ref. [28], is that the initial value

$$
O m(0)=\left.\frac{2}{3} H^{\prime}\right|_{0}=\frac{2\left(8 f_{\mathrm{v} 0}^{3}-3 f_{\mathrm{v} 0}^{2}+4\right)\left(2+f_{\mathrm{v} 0}\right)}{\left(4 f_{\mathrm{v} 0}^{2}+f_{\mathrm{v} 0}+4\right)^{2}}
$$

is substantially larger than in the spatially flat dark energy models. Furthermore, for the timescape model $\mathrm{Om}(z)$ does not asymptote to the dressed density parameter $\Omega_{M 0}$ in any redshift range. For quintessence models $\operatorname{Om}(z)>\Omega_{M 0}$, while for 

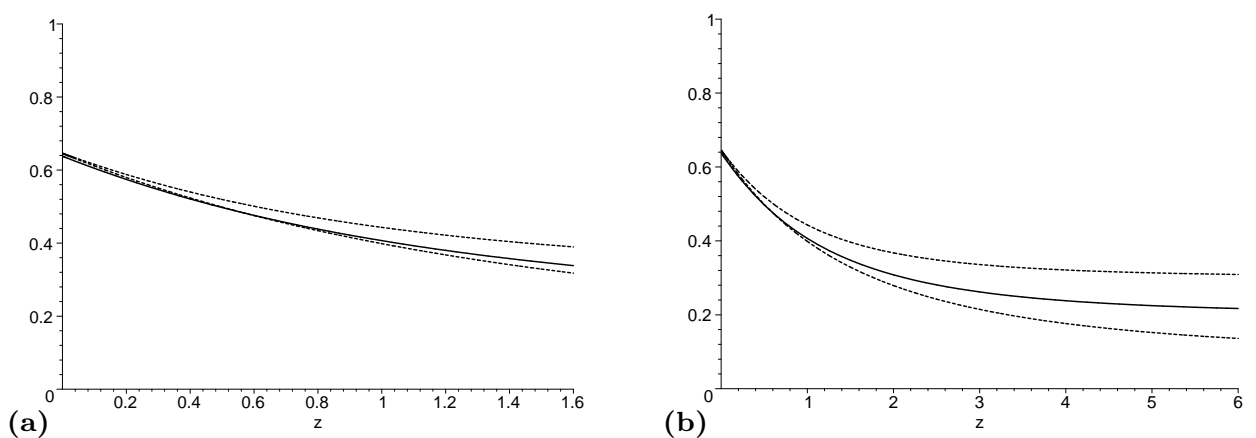

Fig. 5. The dark energy diagnostic $O m(z)$ of Sahni, Shafieloo and Starobinsky ${ }^{28}$ plotted for the timescape tracker solution with best-fit value $f_{\mathrm{v} 0}=0.762$ (solid line), and $1 \sigma$ limits (dashed lines) from ref. [17]: (a) for the redshift range $0<z<1.6$ as shown in ref. [30]; (b) for the redshift range $0<z<6$.

phantom models $\operatorname{Om}(z)<\Omega_{M 0}$, and in both cases $\operatorname{Om}(z) \rightarrow \Omega_{M 0}$ as $z \rightarrow \infty$. In the timescape model, $\operatorname{Om}(z)>\Omega_{M 0} \simeq 0.33$ for $z \lesssim 1.7$, while $\operatorname{Om}(z)<\Omega_{M 0}$ for $z \gtrsim 1.7$. It thus behaves more like a quintessence model for low $z$, in accordance with Fig. 3. However, the steeper slope and the different large $z$ behaviour mean the diagnostic is generally very different to that of typical dark energy models. For large $z, \bar{\Omega}_{M 0}<O m(\infty)<\Omega_{M 0}$, if $f_{\mathrm{v} 0}>0.25$.

Interestingly enough, a recent analysis of SneIa, $\mathrm{BAO}$ and $\mathrm{CMB}$ data ${ }^{30}$ for dark energy models with two different empirical fitting functions for $w(z)$ gives an intercept $\operatorname{Om}(0)$ which is larger than expected for typical quintessence or phantom energy models, and in the better fit of the two models the intercept (see Fig. 3 of ref. [30)] is close to the value expected for the timescape model, which is tightly constrained to the range $0.638<O m(0)<0.646$ if $f_{\mathrm{v} 0}=0.76_{-0.09}^{+0.12}$.

\subsection{The Alcock-Paczyński test and baryon acoustic oscillations}

Some time ago Alcock and Paczyński devised a test ${ }^{31}$ which relies on comparing the radial and transverse proper length scales of spherical standard volumes comoving with the Hubble flow. This test, which determines the function

$$
f_{\mathrm{AP}}=\frac{1}{z}\left|\frac{\delta \theta}{\delta z}\right|=\frac{H D}{z},
$$

was originally conceived to distinguish FLRW models with a cosmological constant from those without a $\Lambda$ term. The test is free from many evolutionary effects, but relies on one being able to remove systematic distortions due to peculiar velocities.

Current detections of the BAO scale in galaxy clustering statistics ${ }^{32,33}$ can in fact be viewed as a variant of the Alcock-Paczyński test, as they make use of both the transverse and radial dilations of the fiducial comoving BAO scale to present a measure

$$
D_{V}=\left[\frac{z D^{2}}{H(z)}\right]^{1 / 3}=D f_{\mathrm{AP}}^{-1 / 3} .
$$



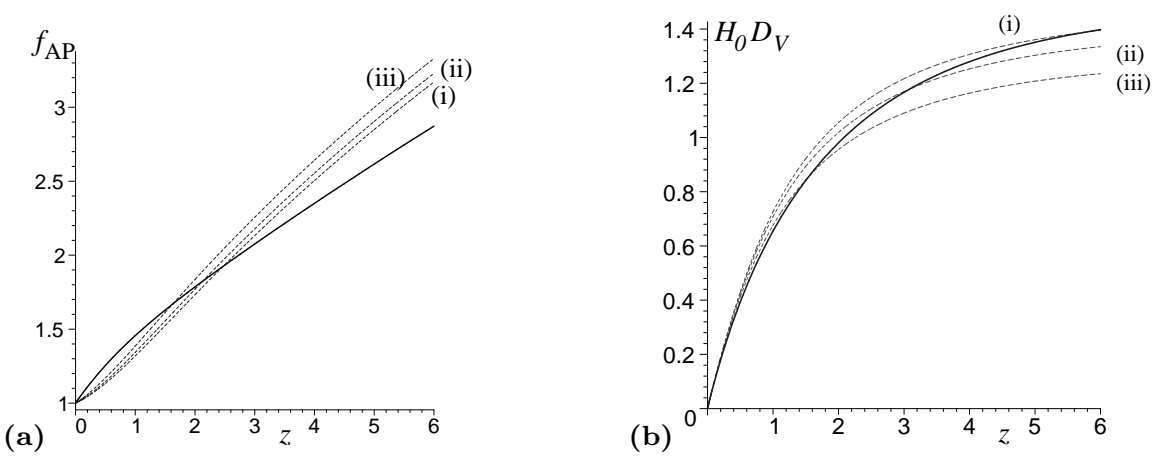

Fig. 6. (a) The Alcock-Paczyński test function $f_{\mathrm{AP}}=H D / z$; and (b) the $\mathrm{BAO}$ radial test function $H_{0} D_{V}=H_{0} D f_{\mathrm{AP}}^{-1 / 3}$. In each case the timescape model with $f_{\mathrm{v} 0}=0.762$ (solid line) is compared to three spatially flat $\Lambda \mathrm{CDM}$ models with the same values of $\left(\Omega_{M 0}, \Omega_{\Lambda 0}\right)$ as in Fig. 2 (dashed lines).

In Fig. 6 the Alcock-Paczyński test function (14) and BAO scale measure (15) of the timescape model are compared to those of the spatially flat $\Lambda$ CDM model with different values of $\left(\Omega_{\Lambda 0}, \Omega_{\Lambda 0}\right)$. Over the range of redshifts $z<1$ studied currently with galaxy clustering statistics, the $f_{\mathrm{AP}}$ curve distinguishes the timescape model from the $\Lambda \mathrm{CDM}$ models much more strongly than the $D_{V}$ test function. In particular, the timescape $f_{\mathrm{AP}}$ has a distinctly different shape to that of the $\Lambda \mathrm{CDM}$ model, being convex. The primary reason for use of the integral measure (15) has been a lack of data. Future measurements with enough data to separate the radial and angular BAO scales are a potentially powerful way of distinguishing the timescape model from $\Lambda$ CDM.

Recently Gaztañaga, Cabré and $\mathrm{Hui}^{26}$ have made the first efforts to separate the radial and angular BAO scales in different redshift slices. Although they have not yet published separate values for the radial and angular scales, their results are interesting when compared to the expectations of the timescape model. Their study yields best-fit values of the present total matter and baryonic matter density parameters, $\Omega_{M 0}$ and $\Omega_{B 0}$, which are in tension with WMAP5 parameters fit to the $\Lambda \mathrm{CDM}$ model. In particular, the ratio of non-baryonic cold dark matter to baryonic matter has a best-fit value $\Omega_{C 0} / \Omega_{B 0}=\left(\Omega_{M 0}-\Omega_{B 0}\right) / \Omega_{B 0}$ of 3.7 in the $0.15<z<0.3$ sample, 2.6 in the $0.4<z<0.47$ sample, and 3.6 in the whole sample, as compared to the expected value of 6.1 from WMAP5. The analysis of the 3-point correlation function yields similar conclusions, with a best fit ${ }^{34} \Omega_{M 0}=0.28 \pm 0.05$, $\Omega_{B 0}=0.079 \pm 0.025$. By comparison, the parameter fit to the timescape model of ref. [17] yields dressed parameters $\Omega_{M 0}=0.33_{-0.16}^{+0.11}, \Omega_{B 0}=0.080_{-0.013}^{+0.021}$, and a ratio $\Omega_{C 0} / \Omega_{B 0}=3.1_{-2.4}^{+2.5}$. Since other forms of dark energy are not generally expected to give rise to a renormalization of the ratio of non-baryonic to baryonic matter, this is encouraging for the timescape model. 


\subsection{Test of (in)homogeneity}

Recently Clarkson, Bassett and $\mathrm{Lu}^{35}$ have constructed what they call a "test of the Copernican principle" based on the observation that for homogeneous, isotropic models which obey the Friedmann equation, the present epoch curvature parameter, a constant, may be written as

$$
\Omega_{k 0}=\frac{\left[H(z) D^{\prime}(z)\right]^{2}-1}{\left[H_{0} D(z)\right]^{2}}
$$

for all $z$, irrespective of the dark energy model or any other model parameters. Consequently, taking a further derivative, the quantity

$$
\mathcal{C}(z) \equiv 1+H^{2}\left(D D^{\prime \prime}-D^{\prime 2}\right)+H H^{\prime} D D^{\prime}
$$

must be zero for all redshifts for any FLRW geometry.

A deviation of $\mathcal{C}(z)$ from zero, or of (16) from a constant value, would therefore mean that the assumption of homogeneity is violated. Although this only constitutes a test of the assumption of the Friedmann equation, i.e., of the Cosmological Principle rather than the broader Copernican Principle adopted in ref. [1], the average inhomogeneity will give a clear and distinct prediction of a non-zero $\mathcal{C}(z)$ for the timescape model.

(a)

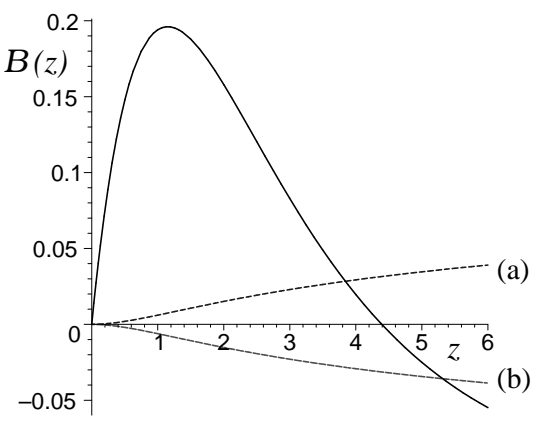

(b)

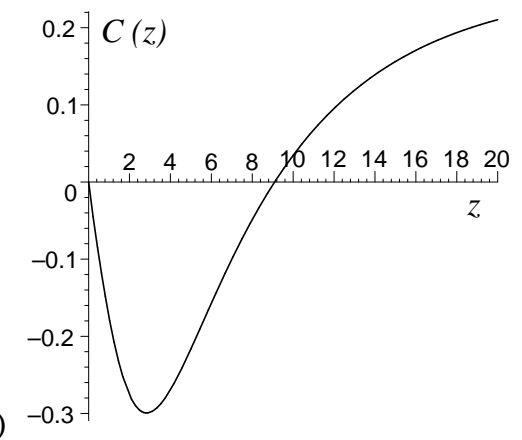

Fig. 7. Left panel: The (in)homogeneity test function $\mathcal{B}(z)=\left[H D^{\prime}\right]^{2}-1$ is plotted for the timescape tracker solution with best-fit value $f_{\mathrm{v} 0}=0.762$ (solid line), and compared to the equivalent curves $\mathcal{B}=\Omega_{k 0}\left(H_{0} D\right)^{2}$ for two different $\Lambda$ CDM models with small curvature: (a) $\Omega_{M 0}=0.28, \Omega_{\Lambda 0}=0.71, \Omega_{k 0}=0.01$; (b) $\Omega_{M 0}=0.28, \Omega_{\Lambda 0}=0.73, \Omega_{k 0}=-0.01$.

Right panel: The (in)homogeneity test function $\mathcal{C}(z)$ is plotted for the $f_{\mathrm{v} 0}=0.762$ tracker solution.

The functions (16) and (17) are computed in ref. [3]. Observationally it is more feasible to fit (16) which involves one derivative less of redshift. In Fig. 7 we exhibit both $\mathcal{C}(z)$, and also the function $\mathcal{B}(z)=\left[H D^{\prime}\right]^{2}-1$ from the numerator of $(16)$ for the timescape model, as compared to two $\Lambda$ CDM models with a small amount of spatial curvature. A spatially flat FLRW model would have $\mathcal{B}(z) \equiv 0$. In other FLRW cases $\mathcal{B}(z)$ is always a monotonic function whose sign is determined by that of 
$\Omega_{k 0}$. An open $\Lambda=0$ universe with the same $\Omega_{M 0}$ would have a monotonic function $\mathcal{B}(z)$ very much greater than that of the timescape model.

\subsection{Time drift of cosmological redshifts}

For the purpose of the $O m(z)$ and (in)homogeneity tests considered in the last section, $H(z)$ must be observationally determined, and this is difficult to achieve in a model independent way. There is one way of achieving this, however, namely by measuring the time variation of the redshifts of different sources over a sufficiently long time interval, ${ }^{36}$ as has been discussed recently by Uzan, Clarkson and Ellis. ${ }^{37}$ Although the measurement is extremely challenging, it may be feasible over a 20 year period by precision measurements of the Lyman- $\alpha$ forest in the redshift range $2<z<5$ with the next generation of Extremely Large Telescopes. ${ }^{38}$

In ref. [3] an analytic expression for $H_{0}^{-1} \frac{\mathrm{d} z}{\mathrm{~d} \tau}$ is determined, the derivative being with respect to wall time for observers in galaxies. The resulting function is displayed in Fig. 8 for the best-fit timescape model with $f_{\mathrm{v} 0}=0.762$, where it is compared to the equivalent function for three different spatially flat $\Lambda \mathrm{CDM}$ models. What is notable is that the curve for the timescape model is considerably flatter than those of the $\Lambda$ CDM models. This may be understood to arise from the fact that the magnitude of the apparent acceleration is considerably smaller in the timescape model, as compared to the magnitude of the acceleration in $\Lambda$ CDM models. For models in which there is no apparent acceleration whatsoever, one finds that $H_{0}^{-1} \frac{\mathrm{d} z}{\mathrm{~d} \tau}$ is always negative. If there is cosmic acceleration, real or apparent, at late epochs then $H_{0}^{-1} \frac{\mathrm{d} z}{\mathrm{~d} \tau}$ will become positive at low redshifts, though at a somewhat larger redshift than that at which acceleration is deemed to have begun.

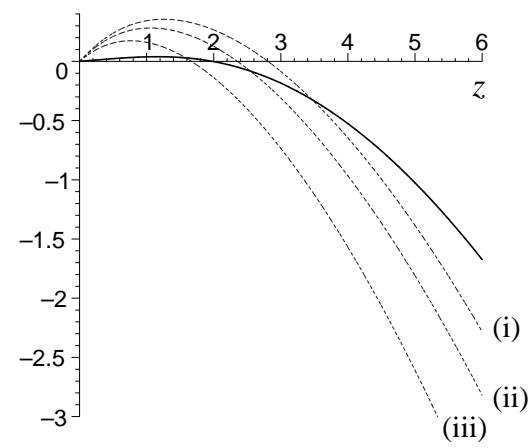

Fig. 8. The function $H_{0}^{-1} \frac{\mathrm{d} z}{\mathrm{~d} \tau}$ for the timescape model with $f_{\mathrm{v} 0}=0.762$ (solid line) is compared to $H_{0}^{-1} \frac{\mathrm{d} z}{\mathrm{~d} \tau}$ for three spatially flat $\Lambda \mathrm{CDM}$ models with the same values of $\left(\Omega_{M 0}, \Omega_{\Lambda 0}\right)$ as in Fig. 2 (dashed lines).

Fig. 8 demonstrates that a very clear signal of differences in the redshift time drift between the timescape model and $\Lambda$ CDM models might be determined at low redshifts when $H_{0}^{-1} \frac{\mathrm{d} z}{\mathrm{~d} \tau}$ should be positive. In particular, the magnitude of $H_{0}^{-1} \frac{\mathrm{d} z}{\mathrm{~d} \tau}$ is 
considerably smaller for the timescape model as compared to $\Lambda$ CDM models. Observationally, however, it is expected that measurements will be best determined for sources in the Lyman $\alpha$ forest in the range, $2<z<5$. At such redshifts the magnitude of the drift is somewhat more pronounced in the case of the $\Lambda$ CDM models. For a source at $z=4$, over a period of $\delta \tau=10$ years we would have $\delta z=-3.3 \times 10^{-10}$ for the timescape model with $f_{\mathrm{v} 0}=0.762$ and $H_{0}=61.7 \mathrm{~km} \mathrm{sec}^{-1} \mathrm{Mpc}^{-1}$. By comparison, for a spatially flat $\Lambda$ CDM model with $H_{0}=70.5 \mathrm{~km} \mathrm{sec}^{-1} \mathrm{Mpc}^{-1}$ a source at $z=4$ would over ten years give $\delta z=-4.7 \times 10^{-10}$ for $\left(\Omega_{M 0}, \Omega_{\Lambda 0}\right)=(0.249,0.751)$, and $\delta z=-7.0 \times 10^{-10}$ for $\left(\Omega_{M 0}, \Omega_{\Lambda 0}\right)=(0.279,0.721)$.

\section{Discussion}

The tests outlined here demonstrate several lines of investigation to distinguish the timescape model from models of homogeneous dark energy. The (in)homogeneity test of Clarkson, Bassett and $\mathrm{Lu}$ is definitive, since it tests the validity of the Friedmann equation directly.

In performing these tests, however, one must be very careful to ensure that data has not been reduced with built-in assumptions that use the Friedmann equation. For example, current estimates of the BAO scale such as that of Percival et $a l .{ }^{33}$ do not determine $D_{V}$ directly from redshift and angular diameter measures, but first perform a Fourier space transformation to a power spectrum, assuming a FLRW cosmology. Redoing such analyses for the timescape model may involve a recalibration of relevant transfer functions.

In the case of supernovae, one must also take care as compilations such as the Union $^{39}$ and Constitution ${ }^{40}$ datasets use the SALT method to calibrate light curves. In this approach empirical light curve parameters and cosmological parameters - assuming the Friedmann equation - are simultaneously fit by analytic marginalisation before the raw apparent magnitudes are recalibrated. As Hicken et al. discuss, ${ }^{40}$ a number of systematic discrepancies exist between data reduced by the SALT, SALT2, MLCS31 and MLCS17 techniques even within the $\Lambda$ CDM model. In the case of the timescape model, we find considerable differences between the different approaches. ${ }^{41}$ In principle, at present there appear to be enough supernovae to decide between the $\Lambda \mathrm{CDM}$ and timescape models on Bayesian evidence, but one is led to different conclusions depending on how the data is reduced. It is therefore important that the systematic issues are unravelled.

The value of the dressed Hubble constant is also an observable quantity of considerable interest. A recent determination of $H_{0}$ by Riess et $a l^{42}$ poses a challenge for the timescape model. However, it is a feature of the timescape model that a $17-22 \%$ variance in the apparent Hubble flow will exist on local scales below the scale of statistical homogeneity, and this may potentially complicate calibration of the cosmic distance ladder. Further quantification of the variance in the apparent Hubble flow in relationship to local cosmic structures would provide an interesting possibility for tests of the timescape cosmology for which there are no counterparts 
in the standard cosmology.

A huge amount of work remains to be done to develop the timescape scenario to the level of detail of the standard cosmology. At the mathematical level, we need to refine the notion of coarse-graining of dust in relation to the various scales of averaging, slicings by hypersurfaces in the evolution equations, and null cone averages. Whatever the outcome of such investigations, I believe that it is exciting that much remains to be still explored in general relativity. The difficult problem of quasi-local gravitational energy in Einstein's theory may turn out not to simply be an arcane curiosity in mathematical relativity, but to be of direct importance for understanding the large scale structure of the universe.

\section{Acknowledgments}

I thank Prof. Remo Ruffini and ICRANet for support and hospitality while the work of refs. $[2,3]$ was undertaken. This work was also partly supported by the Marsden fund of the Royal Society of New Zealand.

\section{References}

1. D. L. Wiltshire, New J. Phys. 9, 377 (2007).

2. D. L. Wiltshire, Phys. Rev. D78, 084032 (2008).

3. D. L. Wiltshire, Phys. Rev. D80, 123512 (2009).

4. D. L. Wiltshire, Phys. Rev. Lett. 99, 251101 (2007).

5. D. L. Wiltshire, in Dark Matter in Astroparticle and Particle Physics: Proc. of the 6th International Heidelberg Conference, eds H. V. Klapdor-Kleingrothaus and G. F. Lewis, (World Scientific, Singapore, 2008) pp. 565-596 [arXiv:0712.3984].

6. F. Hoyle and M. S. Vogeley, Astrophys. J. 566, 641 (2002); Astrophys. J. 607, 751 (2004).

7. K. Bolejko, A. Krasiński, C. Hellaby and M.-N. Célérier, Structures in the Universe by Exact Methods, (Cambridge University Press, 2009)

8. G. Lemaître, Ann. Soc. Sci. Bruxelles A 53 (1933) 51 [English translation in Gen. Relativ. Grav. 29 (1997) 641]; R.C. Tolman, Proc. Nat. Acad. Sci. 20 (1934) 169; H. Bondi, Mon. Not. R. Astr. Soc. 107 (1947) 410.

9. R. J. van den Hoogen, in this volume; arXiv:1003.4020.

10. T. Buchert, Gen. Relativ. Grav. 32, 105 (2000); Gen. Relativ. Grav. 33, 1381 (2001).

11. T. Buchert, Gen. Relativ. Grav. 40, 467 (2008).

12. P. J. E. Peebles, arXiv:0910.5142.

13. L.B. Szabados, Living Rev. Rel. 7, 4 (2004).

14. A. Einstein, Sitzungsber. Preuss. Akad. Wiss., (1917) 142 [English translation in "The collected papers of Albert Einstein. Vol. 6" (Princeton Univ. Press, 1997) pp. 421-432].

15. H. Bondi, Cosmology, (Cambridge Univ. Press, 1961)

16. J. Bičák, J. Katz and D. Lynden-Bell, Phys. Rev. D 76, 063501 (2007).

17. B. M. Leith, S. C. C. Ng and D. L. Wiltshire, Astrophys. J. 672, L91 (2008).

18. S. Jha, A. G. Riess and R. P. Kirshner, Astrophys. J. 659, 122 (2007).

19. N. Li and D. J. Schwarz, Phys. Rev. D78, 083531 (2008).

20. E. Komatsu et al., Astrophys. J. Suppl. 180, 330 (2009).

21. A. G. Riess et al., Astrophys. J. 659, 98 (2007).

22. B. E. Schaefer, Astrophys. J. 660, 16 (2007); N. Liang, W. K. Xiao, Y. Liu and S. N. Zhang, Astrophys. J. 685, 354 (2008). L. Amati, C. Guidorzi, F. Frontera, M. Della 
Valle, F. Finelli, R. Landi and E. Montanari, Mon. Not. R. Astr. Soc. 391, 577 (2008); R. Tsutsui, T. Nakamura, D. Yonetoku, T. Murakami, Y. Kodama and K. Takahashi, JCAP 08 (2009) 015.

23. B. E. Schaefer, talk at Sixth Huntsville Gamma Ray Burst Symposium 2008, http://grbhuntsville2008.cspar.uah.edu/content/Talks/Schaefer.pdf

24. G. B. Zhao and X. Zhang, Phys. Rev. D81, 043518 (2010).

25. P. Serra, A. Cooray, D. E. Holz, A. Melchiorri, S. Pandolfi and D. Sarkar, Phys. Rev. D80, 121302 (2009).

26. E. Gaztañaga, A. Cabre and L. Hui, Mon. Not. R. Astr. Soc. 399, 1663 (2009).

27. J. A. Gu, C. W. Chen and P. Chen, New J. Phys. 11, 073029 (2009).

28. V. Sahni, A. Shafieloo and A. A. Starobinsky, Phys. Rev. D78, 103502 (2008).

29. C. Zunckel and C. Clarkson, Phys. Rev. Lett. 101, 181301 (2008).

30. A. Shafieloo, V. Sahni and A. A. Starobinsky, Phys. Rev. D80, 101301 (2009).

31. C. Alcock and B. Paczyński, Nature 281, 358 (1979).

32. D. J. Eisenstein et al., Astrophys. J. 633 (2005) 560; S. Cole et al., Mon. Not. R. Astr. Soc. 362 (2005) 505.

33. W. J. Percival et al., Mon. Not. R. Astr. Soc. 381, 1053 (2007); W. J. Percival et al., Mon. Not. R. Astr. Soc. 401, 2148 (2010).

34. E. Gaztañaga, A. Cabré, F. Castander, M. Crocce and P. Fosalba, Mon. Not. R. Astr. Soc. 399, 801 (2009).

35. C. Clarkson, B. Bassett and T. C. Lu, Phys. Rev. Lett. 101, 011301 (2008).

36. A. Sandage, Astrophys. J. 136, 319 (1962); G. C. McVittie, Astrophys. J. 136, 334 (1962); A. Loeb, Astrophys. J. 499, L111 (1998).

37. J. P. Uzan, C. Clarkson and G. F. R. Ellis, Phys. Rev. Lett. 100, 191303 (2008).

38. P. S. Corasaniti, D. Huterer and A. Melchiorri, Phys. Rev. D75, 062001 (2007); J. Liske et al., Mon. Not. R. Astr. Soc. 386, 1192 (2008).

39. M. Kowalski et al., Astrophys. J. 686, 749 (2008).

40. M. Hicken et al., Astrophys. J. 700, 1097 (2009).

41. P. R. Smale and D. L. Wiltshire, in preparation.

42. A. G. Riess et al., Astrophys. J. 699, 539 (2009). 